\title{
Tumor-associated fibroblast-conditioned medium promotes tumor cell proliferation and angiogenesis
}

\author{
L.N. Xu' ${ }^{1}$ B.N. Xu' ${ }^{2}$, J. Cai ${ }^{1}$, J.B. Yang ${ }^{1}$ and N. $\operatorname{Lin}^{3}$ \\ ${ }^{1}$ Department of Gynecology and Obstetrics, \\ The Sixth Affiliate Hospital of Sun Yet-Sen University, Guangzhou, China \\ ${ }^{2}$ Department of Gynecology and Obstetrics, The First People's Hospital, \\ Foshan, China \\ ${ }^{3}$ Department of Hepatobiliary Surgery, \\ The Third Affiliate Hospital of Sun Yet-Sen University, Guangzhou, China \\ Corresponding author: N. Lin \\ E-mail: nanlincn@126.com
}

Genet. Mol. Res. 12 (4): 5863-5871 (2013)

Received March 8, 2013

Accepted September 2, 2013

Published November 22, 2013

DOI http://dx.doi.org/10.4238/2013.November.22.14

\begin{abstract}
This study aimed to explore how tumor-associated fibroblasts (TAFs) promote the proliferation and angiogenesis of tumor cells via the paracrine mechanism in vitro. Conditioned media (CM) of ovarian TAFs and normal fibroblasts (NFs) were collected. Ovarian cancer cells (OCCs) were treated with $2 \mathrm{~mL}$ TAFs-CM and NFs-CM in experimental and control groups, respectively; $20 \mu \mathrm{M}$ SB431512, a specific small molecule inhibitor of transforming growth factor- $\beta$ (TGF- $\beta$ ), was added in the experimental group as the intervention group. The cell cycle was determined in each group. mRNA expressions of proliferating cell nuclear antigen (PCNA), $\alpha$-smooth muscle actin $(\alpha-S M A)$, and vascular endothelial growth factor (VEGF), and protein expressions of $\alpha$-SMA and VEGF were detected in each group. Proliferation of OCCs was significantly promoted in the experimental group compared with that of the control group. The proliferative
\end{abstract}


effect was obviously inhibited in the intervention group. The mRNA expressions of PCNA, $\alpha$-SMA, and VEGF, and protein expressions of $\alpha$-SMA and VEGF were all dramatically up-regulated in each group, and were strongly inhibited by SB-431512. TAFs promote the proliferation of OCCs via paracrine and up-regulated expression of angiogenic genes and proteins, which can be effectively inhibited by inhibiting the TGF- $\beta$ signaling pathway.

Key words: Tumor-associated fibroblasts; Ovarian cancer cells; Transforming growth factor- $\beta$

\section{INTRODUCTION}

Recent research has discovered that the tumor microenvironment, which is composed of stromal cells and extracellular matrix components, plays an important role in tumor progression (Galié et al., 2005). Tumor-associated fibroblasts (TAFs) are crucial members of the tumor microenvironment, and are considered to promote the initiation, growth, invasion, and angiogenesis of tumors via multiple mechanisms in various organs, including the breast, pancreas, and ovary (Liotta and Kohn, 2001; Sonnenberg et al., 2008; Xing et al., 2010; Cirri and Chiarugi, 2012). TAFs interact with tumor cells through the large numbers of cytokines that are secreted in a paracrine manner and that regulate their biological activities (Raman et al., 2007). Among these cytokines, transforming growth factor- $\beta$ (TGF- $\beta$ ) is secreted by TAFs, and is thus important for the growth of tumor cells (Narine et al., 2004; Fuyuhiro et al., 2011). It also causes immunosuppression and angiogenesis in the tumor microenvironment, which makes the cancer more invasive (Blobe et al., 2000).

Ovarian cancer is a female malignant tumor that is common worldwide. The five-year survival rate for this fatal disease is very low due to its high rate of recurrence and metastasis (Cannistra, 2004). Thus, there is an urgent need to advance our understanding of the mechanism of ovarian cancer progression.

Among ovarian cancer stroma, TAFs are the most important cells influencing the initiation and development of ovarian cancer (Parrott et al., 2001). In vivo experiments revealed that TAFs accelerated tumor formation after injecting both TAFs and human ovarian cancer cells (OCCs) with weak tumorigenicity in mice (Terada, 2011), indicating that TAFs may promote tumor growth by particular mechanisms. In colorectal liver metastasis, TAF-derived TGF- $\beta$ promoted tumor growth via a growth-promoting effect on colorectal cancer cells. Our previous study also showed that exogenous TGF- $\beta$ promoted the proliferation rate of OCCs, and other effects of TGF- $\beta$ on OCCs are currently under investigation.

Ovarian cancer, like many other tumors, has a rich blood supply and relies on the formation of blood vessels for growth and metastasis. The formation of new vessels in the tumor is controlled simultaneously by pro- and anti-angiogenic factors. The most important of several pro-angiogenic factors is the vascular endothelial growth factor (VEGF). The upregulation of VEGF expression in tumor tissues has been reported to be associated with poor prognosis in several cancers, including ovarian cancer.

Therefore, we investigated whether TAFs might promote the proliferation and VEGF secretion of OCCs. Up-regulated proliferation and VEGF expression of OCCs could promote angiogenesis in tumor stroma, and eventually facilitate the growth and metastasis of ovarian 
cancer. Our research aimed to evaluate the influence of conditioned media (CM) of TAFs on the proliferation and expression of angiogenic genes and proteins of OCCs. In vitro experiments were also performed to explore whether the paracrine effect of TAFs on OCCs occurred through the TGF- $\beta$ signaling pathway using the TGF- $\beta$ neutralization method.

\section{MATERIAL AND METHODS}

\section{Cell isolation and culture}

Ovarian samples were collected from three patients with epithelial ovarian cancer under aseptic condition in the operating room, from which TAFs were isolated. Normal fibroblasts (NFs) were isolated from normal ovarian samples, which were collected from three patients who underwent radical trachelectomies. All samples were stored in phosphate-buffered solution (PBS) at $4{ }^{\circ} \mathrm{C}$ and related cells were isolated within $4 \mathrm{~h}$.

Samples were washed in PBS containing $100 \mathrm{U} / \mathrm{mL}$ penicillin-streptomycin, the epithelium and adipose tissues were removed, and the residual connective tissue was cut into small pieces ( $2 \times 2 \times 2 \mathrm{~mm}$ ), which were digested in a separating medium (high glucoseDulbecco's modified Eagle's medium (HG-DMEM), 10\% fetal bovine serum (FBS), $0.1 \%$ collagenase I, and $0.1 \%$ hyaluronidase) in an incubator at $37^{\circ} \mathrm{C}$ with $5 \% \mathrm{CO}_{2}$ for 3 days. Subsequently, complete medium (HG-DMEM, 10\% FBS) was used and changed regularly. Cells were cultured with the adherent method to approximately $80-90 \%$ confluence, followed by digestion with $0.25 \%$ trypsin and $0.01 \%$ ethylenediaminetetraacetic acid (EDTA) for 2 min, which was stopped by the addition of $2 \mathrm{~mL}$ serum-supplemented medium, and contraction and refraction enhancement of cell cytoplasm were observed under phase contrast microscopy. The cell suspension was prepared, seeded after counting, and recorded as P1. TAFs were purified from P3 for the establishment of the experimental group and identification. NFs were prepared with the same methods as used for TAF preparation.

\section{Immunofluorescence assay}

Identification of TAFs and NFs was accomplished with an immunofluorescence assay. TAFs specifically expressed $\alpha$-smooth muscle actin ( $\alpha$-SMA), which was used for discrimination between TAFs and NFs. According to conventional immunofluorescence cytochemistry, cells were fixed with $4 \%$ paraformaldehyde for $10 \mathrm{~min}$, and their permeability was increased with $0.3 \%$ Triton for $1 \mathrm{~h}$ following kit instructions. The working concentration of the $\alpha$-SMA antibody was 1:200; PBS was used as the negative control. Cells were incubated with primary antibody overnight at $4^{\circ} \mathrm{C}$, followed by biotin-labeled secondary antibody, enzyme-marked streptavidin peroxidase, and diaminobenzidine development. Red fluorescence in cells was considered as a positive signal.

\section{Collection of TAFs-CM and NFs-CM}

CM was collected when the confluence of TAFs and NFs reached $80-90 \%$, and then centrifugation was performed at $12,000 \mathrm{~g}$ at $4^{\circ} \mathrm{C}$ for $10 \mathrm{~min}$ to remove cell debris, followed by filtration with a $0.22-\mu \mathrm{m}$ filter. The $\mathrm{CM}$ was preserved at $-80^{\circ} \mathrm{C}$ for further study. 
SKOV-3 cells were seeded on a six-well plate at $1 \times 10^{5}$ cells/well. Serum-free medium was added to all wells on the first day. On the second day, experimental, control, blank, and intervention groups were treated with $2 \mathrm{~mL}$ TAFs-CM, NFs-CM, complete medium, and 0.01 M SB-431542 (a specific small molecule inhibitor of TGF- $\beta$ ), respectively.

\section{Flow cytometry}

After a 48-h incubation with CM, OCCs were digested with $0.25 \%$ trypsin, washed with PBS, and fixed in $70 \%$ pre-cooled ethanol overnight at $4^{\circ} \mathrm{C}$. The washing step was repeated twice with equivalent PBS volumes, and then $100 \mu \mathrm{L}$ RNase A was added for incubation at $37^{\circ} \mathrm{C}$ in a water bath for $30 \mathrm{~min}$. Propidium iodide was utilized for color development at $4^{\circ} \mathrm{C}$ for 30 min, followed by flow cytometry detection and cell cycle analysis with the WinMD software.

\section{Reverse transcription-polymerase chain reaction (RT-PCR)}

Analysis of mRNA expressions of proliferating cell nuclear antigen, $\alpha$-SMA, and VEGF was accomplished with RT-PCR. After a 48-h incubation with CM, OCCs were washed with PBS once after removal of CM. One milliliter RNAiso Plus was added to each well. The cell layer was dissolved by gently beating and sucking into an Eppendorf tube to stand at room temperature for $5 \mathrm{~min}$, and was then mixed with $200 \mu \mathrm{L}$ chloroform for $5 \mathrm{~min}$, followed by centrifugation at $12,000 \mathrm{~g}$ at $4^{\circ} \mathrm{C}$ for $15 \mathrm{~min}$. The centrifuge tube was carefully taken out, and the homogenate showed three layers; the upper suspension was transferred to a new Eppendorf tube and mixed with $500 \mu \mathrm{L}$ propofol for $10 \mathrm{~min}$. Centrifugation was performed at $12,000 \mathrm{~g}$ at $4^{\circ} \mathrm{C}$ for $10 \mathrm{~min}$, and the suspension was removed carefully. One milliliter $75 \%$ ethanol was used to wash the tube, followed by another centrifugation at $12,000 \mathrm{~g}$ at $4^{\circ} \mathrm{C}$ for $5 \mathrm{~min}$. The pellet was dried at room temperature for $5 \mathrm{~min}$, dissolved in $40 \mu \mathrm{L}$ inactivated RNase water, and stored at $-80^{\circ} \mathrm{C}$. Twenty microliters cDNA was synthesized with RT-PCR using extracted total RNA as samples. A $20-\mu \mathrm{L}$ reaction model was established as follows: $2 \mu \mathrm{L}$ cDNA, $2 \mu \mathrm{L}$ forward primer, $2 \mu \mathrm{L}$ reverse primer, $5 \mu \mathrm{L} 2 \mathrm{X} \mathrm{HS}^{\mathrm{TM}}$ reaction mixture, $0.2 \mu \mathrm{L} 2.5 \mathrm{U} / \mu \mathrm{L}$ DNA polymerase, and $8.8 \mu \mathrm{L}$ ultrapure water. All steps were performed on ice. The PCR amplification conditions were as follows: $94^{\circ} \mathrm{C}$ for $3 \mathrm{~min}, 94^{\circ} \mathrm{C}$ for $30 \mathrm{~s}$ for 30 cycles, $60^{\circ} \mathrm{C}$ for $30 \mathrm{~s}$ for 30 cycles, $72^{\circ} \mathrm{C}$ for $1 \mathrm{~min}$ for $30 \mathrm{cycles}$, and $72^{\circ} \mathrm{C}$ for $10 \mathrm{~min}$. Five microliters amplified solution was loaded on $1 \%$ agarose gel for detection. The gray scale of the electrophoresis strip was scanned using an ultraviolet photometry gel imaging system, and was analyzed with software. The results were presented as the gray scale ratio between the target gene and the internal reference gene. The experiment was repeated three times. The designed primers are shown in Table 1.

\begin{tabular}{ll} 
Table 1. Primers of target gene in PCR. & \\
\hline Gene & Sequence of primers \\
\hline PCNA & F 5'-AAACTAGCTAGACTTTCCTC-3' \\
& R 5'-TCACGCCCATGGCCAGGTTG-3' \\
& F 5'-AGGAAGGACCTCTATGCTAACAAT-3' \\
VEGF & R 5'-AACACATAGGTAACGAGTCAGAGC-3' \\
$\beta$-Actin & F 5'-TGCATTCACATTTGTTGTGC-3' \\
& R 5'-AGACCCTGGTGGACATCTTC-3' \\
& F 5'-TCACCCACACTGTGCCCATCTACGA-3' \\
\hline
\end{tabular}




\section{Western blot (WB)}

Detection of TGF- $\beta 1, \alpha$-SMA, and VEGF expressions was accomplished with WB analysis. After a 48-h incubation with CM, OCCs were collected and lysed in cell lysis buffer. Total protein was extracted and quantified using the Bradford method. Electrophoresis was performed with $80 \mu \mathrm{g}$ total protein loaded on $10 \%$ polyacrylamide gel, and then the protein on gel was transferred to a polyvinylidene fluoride membrane, which was blocked with $5 \%$ bovine serum albumin and Tris-buffered saline with Tween. The membrane was incubated overnight at $4^{\circ} \mathrm{C}$ with the following primary antibodies: rabbitanti-human TGF- $\beta 1, \alpha$-SMA, and VEGF monoclonal antibody. After washing, horseradish peroxidase-labeled secondary antibody was added, and color was developed for 1-5 min with enhanced chemiluminescent substrate. The gray scale of the electrophoresis strip was detected with the ImageJ analysis software. The corresponding values from deep to shallow color were 0 (black) to 250 (white). For a more intuitive comparison, corrected values were obtained by subtracting the measured value from 250 . The experiment was repeated three times.

\section{Statistical analysis}

Measurement data are reported as means \pm standard deviation, and were analyzed with the SPSS17.0 software. The Student $t$-test was used for comparisons between two groups.

\section{RESULTS}

\section{Morphological observations of TAFs and NFs and their identification by immunofluorescence}

TAFs appeared as long spindle shapes with little cytoplasm, which grew fast and desultorily. Contact inhibition was rare, even when the cell density was high. NFs appeared as flat star shapes with a substantial amount of cytoplasm, which grew slowly in the same size and formed clusters. Contact inhibition was observed when the cell density was high. The immunofluorescence assay showed a relatively high expression of $\alpha$-SMA in TAFs (red under a fluorescence microscope) compared to NFs (data not shown).

\section{TAFs-CM significantly promoted SKOV-3 proliferation}

Compared with the control group, the experimental group showed obviously increased proliferation of SKOV-3 cells; the ratio of cells in the S phase of the cell cycle was significantly higher in the experimental group $(22.10 \pm 1.84)$ compared to the control group $(12.77 \pm 1.43 ; \mathrm{P}<0.05)$. The ability of TAFs to promote SKOV-3 proliferation was markedly inhibited in the intervention group compared with the experimental group; the ratio of $\mathrm{S}$ phase cells in the intervention group $(12.33 \pm 1.12)$ was significantly lower than that of the experimental group $(22.10 \pm 1.84 ; \mathrm{P}<0.05)$, but showed no statistical difference compared with that of the control group $(\mathrm{P}>0.05)$ (Figure 1). 


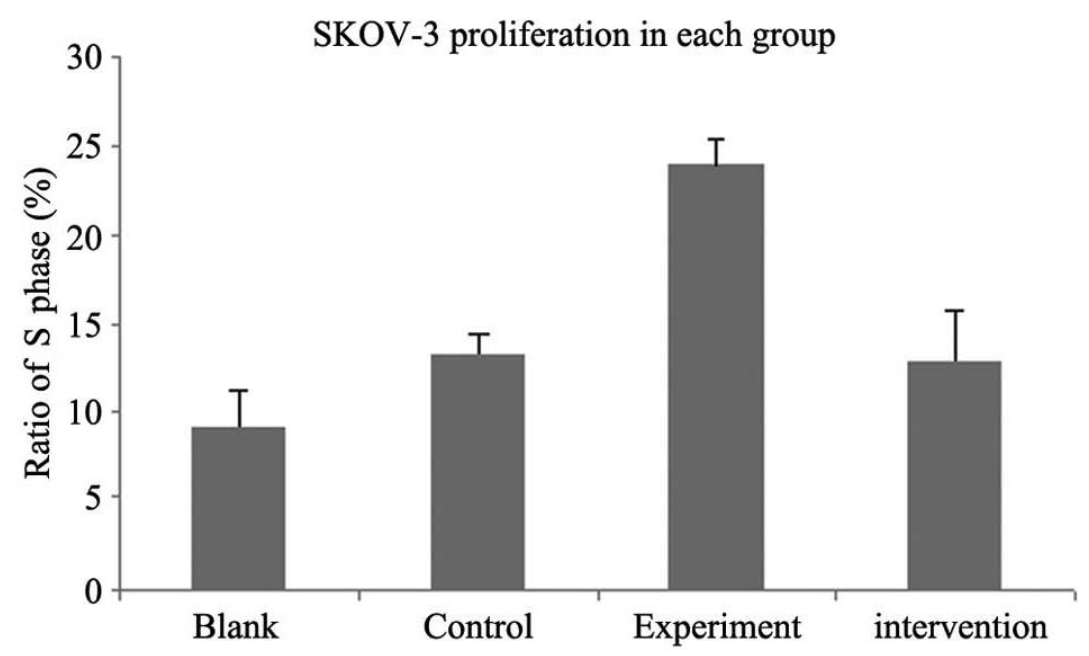

Figure 1. Effect of TAFs-CM on SKOV-3 proliferation.

\section{TAFs-CM up-regulated mRNA expression of PCNA, $\alpha$-SMA, and VEGF in} SKOV-3 cells

RT-PCR revealed that mRNA expression of PCNA was dramatically up-regulated in the experimental group compared with control and blank groups, which was consistent with the previous results indicating that TAFs-CM promoted SKOV-3 proliferation. VEGF and $\alpha$-SMA mRNA expression levels were also increased in the experimental group (Figure 2).

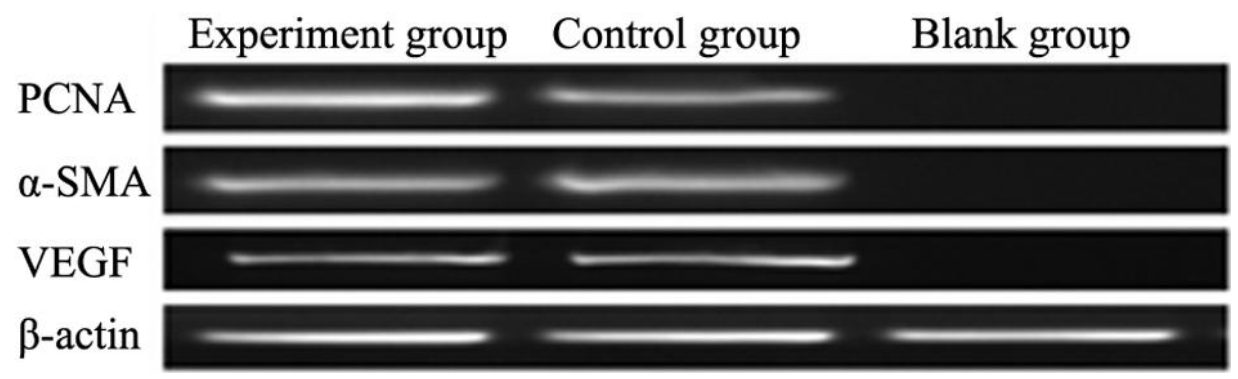

Figure 2. Effect of different CM on mRNA expression of PCNA, $\alpha$-SMA, and VEGF in SKOV-3.

TAFs up-regulated protein expression of $\alpha$-SMA and VEGF in SKOV-3 cells through the TGF- $\beta$ signaling pathway

The WB results showed that protein expressions of $\alpha$-SMA and VEGF in the experimental group were significantly higher than those of the control and blank groups $(\mathrm{P}<0.05)$. Protein expressions of $\alpha$-SMA and VEGF in the intervention group were obviously inhibited, 
which indicated that the expressions of these pro-angiogenic proteins were regulated by SB431512, an inhibitor of the TGF- $\beta$ signaling pathway (Figure 3).

A

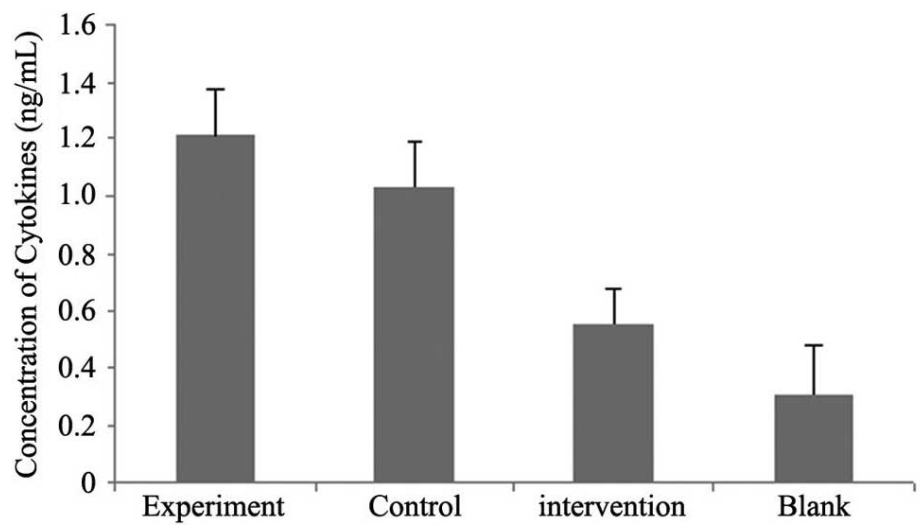

B

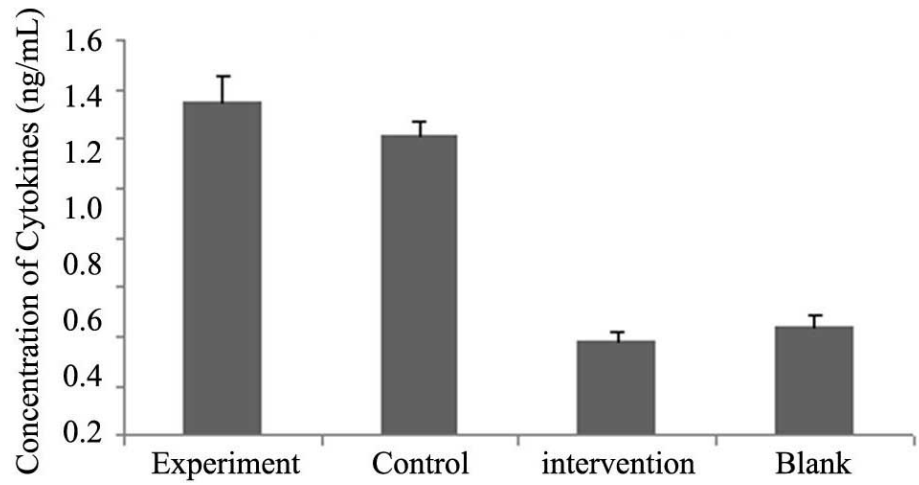

C

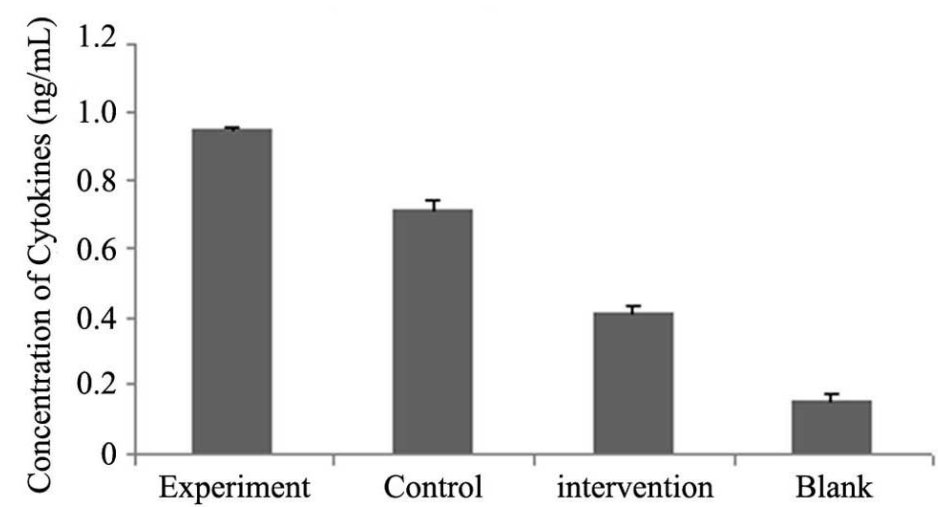

Figure 3. Relevant protein expression in SKOV-3 in each group. A. TGF- $\beta 1$. B. $\alpha$-SMA. C. VEGF. 


\section{DISCUSSION}

Recent research has demonstrated that TAFs have an important supportive effect on tumor cells in the tumor microenvironment, which act to promote tumor cell initiation, growth, invasion, and angiogenesis via multiple mechanisms (Liotta and Kohn, 2001). Our in vitro experiment demonstrated that TAFs-CM-treated OCC lines (SKOV-3) showed elevated proliferation compared with NFs-CM-treated cells. At the same time, the gene and protein expression of angiogenic factors ( $\alpha$-SMA and VEGF) also increased in SKOV-3 cells treated with TAFs-CM, which indicated that TAFs promote the proliferation and proangiogenic effects of OCCs in a paracrine manner. These results explain the supporting effect of TAFs in the stroma of ovarian cancer.

TAFs-CM was confirmed to be composed of multiple cytokines, including TGF- $\beta$, platelet-derived growth factor, basic fibroblast growth factor, and TNF- $\alpha$, compared with NFs-CM (Schmitt-Graff et al., 1994; Jain et al., 2008; Mueller et al., 2010). TGF- $\beta$, a multifunctional cell growth factor, is involved in the regulation of cell proliferation, invasion, differentiation and apoptosis, and is also closely correlated with the initiation and development of many types of tumors (Kiyono et al., 2009; Yu et al., 2010; Drabsch and ten Dijke, 2011). Yan et al. (2002) found that TGF- $\beta$ effectively promoted the proliferation of colon cancer cells through cross-linking of the Ras signaling pathway (instead of the Smad signaling pathway). Gatza et al. (2011) demonstrated that TGF- $\beta$ III receptors were correlated with the mobility of colon cancer cells. In an in vivo animal experiment, tumor size was markedly reduced after inhibiting the function of TGF- $\beta$. The present study used SB431512, a specific small molecule inhibitor of TGF- $\beta$, to block the TGF- $\beta$ signaling pathway. The results showed that the proliferation of OCCs up-regulated by TAFs-CM was dramatically inhibited. This indicates that TGF- $\beta$ also promotes the proliferation of ovarian cancers, as well as other types of cancers.

Tumor progression requires the formation of new blood vessels. Interactions between tumor and stromal cells are important for the abnormal angiogenesis that occurs in tumors (Ribatti and Vacca, 2008). Stromal cells and their secreted factors constitute an appropriate microenvironment for tumor angiogenesis. TAFs, as an important type of stromal cell in ovarian cancer, may participate in tumor angiogenesis (Agarwal et al., 2010; Schmitt and Matei, 2012). We used the TGF- $\beta$ inhibition method to discover that TGF- $\beta$ derived from TAFs mediated the enhanced expression of $\alpha$-SMA and VEGF in OCCs, which might play an important role in the angiogenesis of ovarian cancer. Future in vivo studies should investigate whether inhibiting TGF- $\beta$ expression in TAFs would affect angiogenesis of ovarian cancer and reduce the growth and metastasis of this aggressive tumor.

The present study showed that TAFs had a crucial effect on the proliferation and metastasis of OCCs, which depended on the TGF- $\beta$ signaling pathway. TAFs might secrete TGF- $\beta$ via a paracrine method, thus promoting OCC proliferation in the tumor microenvironment and simultaneously stimulating OCCs to secrete large amounts of VEGF, resulting in angiogenesis in tumors, and thus facilitating OCC proliferation and metastasis. Crosslinking of TGF- $\beta$ and related cytokines between TAFs and OCCs is likely the key factor involved in the occurrence and development of ovarian cancer. Therefore, this should constitute a future therapeutic target for ovarian cancer. 


\section{ACKNOWLEDGMENTS}

Research supported by the National Science Foundation of China (\#81272642), the National Science Foundation for Young Scholars of China (\#81000674), the Natural Science Foundation of Guangdong Province, China (\#S2011010004729), the Fundamental Research Funds for the Central Universities (Sun Yat-Sen University, \#10120110040), and the Science and Technology Planning Project of Guangdong Province, China (\#2010B0316003).

\section{REFERENCES}

Agarwal A, Tressel SL, Kaimal R, Balla M, et al. (2010). Identification of a metalloprotease-chemokine signaling system in the ovarian cancer microenvironment: implications for antiangiogenic therapy. Cancer Res. 70: 5880-5890.

Blobe GC, Schiemann WP and Lodish HF (2000). Role of transforming growth factor beta in human disease. N. Engl. J. Med. 342: 1350-1358.

Cannistra SA (2004). Cancer of the ovary. N. Engl. J. Med. 351: 2519-2529.

Cirri P and Chiarugi P (2012). Cancer-associated-fibroblasts and tumour cells: a diabolic liaison driving cancer progression. Cancer Metastasis Rev. 31: 195-208.

Drabsch Y and ten Dijke P (2011). TGF-beta signaling in breast cancer cell invasion and bone metastasis. J. Mammary Gland Biol. Neoplasia 16: 97-108.

Fuyuhiro Y, Yashiro M, Noda S, Kashiwagi S, et al. (2011). Upregulation of cancer-associated myofibroblasts by TGFbeta from scirrhous gastric carcinoma cells. Br. J. Cancer 105: 996-1001.

Galié M, Sorrention C, Montani M and Micossi L (2005). Mammary carcinoma provides highly tumorigenic and invasive reactive stromal cells. Carcinogenesis 26: 1868-1878.

Gatza CE, Holtzhausen A, Kirkbride KC, Morton A, et al. (2011). Type III TGF-beta receptor enhances colon cancer cell migration and anchorage-independent growth. Neoplasia 13: 758-770.

Jain RK, Lahdenranta J and Fukumura D (2008). Targeting PDGF signaling in carcinoma-associated fibroblasts controls cervical cancer in mouse model. PLoS Med. 5: e24.

Kiyono K, Suzuki HI, Matsuyama H, Morishita Y, et al. (2009). Autophagy is activated by TGF-beta and potentiates TGFbeta-mediated growth inhibition in human hepatocellular carcinoma cells. Cancer Res. 69: 8844-8852.

Liotta LA and Kohn EC (2001). The microenvironment of the tumour-host interface. Nature 411: 375-379.

Mueller L, von Seggern L, Schumacher J, Goumas F, et al. (2010). TNF-alpha similarly induces IL-6 and MCP-1 in fibroblasts from colorectal liver metastases and normal liver fibroblasts. Biochem. Biophys. Res. Commun. 397 : 586-591.

Narine K, DeWever O, Cathenis K, Mareel M, et al. (2004). Transforming growth factor-beta-induced transition of fibroblasts: a model for myofibroblast procurement in tissue valve engineering. J. Heart Valve Dis. 13: 281-289.

Parrott JA, Nilsson E, Mosher R, Magrane G, et al. (2001). Stromal-epithelial interactions in the progression of ovarian cancer: influence and source of tumor stromal cells. Mol. Cell. Endocrinol. 175: 29-39.

Raman D, Baugher PJ, Thu YM and Richmond A (2007). Role of chemokines in tumor growth. Cancer Lett. 256: 137-165.

Ribatti D and Vacca A (2008). The role of microenvironment in tumor angiogenesis. Genes Nutr. 3: 29-34.

Schmitt J and Matei D (2012). Targeting angiogenesis in ovarian cancer. Cancer Treat. Rev. 38: 272-283.

Schmitt-Graff A, Desmouliere A and Gabbiani G (1994). Heterogeneity of myofibroblast phenotypic features: an example of fibroblastic cell plasticity. Virchows Arch. 425: 3-24.

Sonnenberg M, van der Kuip H, Haubeis S, Fritz P, et al. (2008). Highly variable response to cytotoxic chemotherapy in carcinoma-associated fibroblasts (CAFs) from lung and breast. BMC Cancer 8: 364.

Terada T (2011). Ovarian malignant Mullerian mixed tumor (heterologous) whose epithelial component is composed predominantly of signet ring cell carcinoma. Arch. Gynecol. Obstet. 283: 1403-1406.

Xing F, Saidou J and Watabe K (2010). Cancer associated fibroblasts (CAFs) in tumor microenvironment. Front Biosci. 15: 166-179.

Yan Z, Kim GY, Deng X and Friedman E (2002). Transforming growth factor beta 1 induces proliferation in colon carcinoma cells by Ras-dependent, smad-independent down-regulation of p21cip1. J. Biol. Chem. 277: 9870-9879.

Yu N, Kozlowski JM, Park II, Chen L, et al. (2010). Overexpression of transforming growth factor beta1 in malignant prostate cells is partly caused by a runaway of TGF-betal auto-induction mediated through a defective recruitment of protein phosphatase 2A by TGF-beta type I receptor. Urology 76: 1519-13. 